\title{
Towards AC-induced optimum control of dynamical localization
}

\author{
F. Revuelta ${ }^{1,2}$, R. Chacón ${ }^{3,4}$ and F. Borondo ${ }^{2,5}$ \\ 1 Grupo de Sistemas Complejos, Escuela Técnica Superior de Ingenieros Agrónomos, \\ Universidad Politécnica de Madrid - Avda. Complutense s/n, 28040 Madrid, Spain \\ 2 Instituto de Ciencias Matemáticas (ICMAT) - Cantoblanco, 28049 Madrid, Spain \\ 3 Departamento de Física Aplicada, Escuela de Ingenierías Industriales, Universidad de Extremadura \\ Apartado Postal 382, 06006 Badajoz, Spain \\ 4 Instituto de Computación Científica Avanzada, Universidad de Extremadura - Apartado Postal 382, \\ 06006 Badajoz, Spain \\ ${ }^{5}$ Departamento de Química, Universidad Autónoma de Madrid-Cantoblanco, 28049 Madrid, Spain
}

received 27 February 2015; accepted in final form 20 May 2015

published online 12 June 2015

PACS 05.45.Mt - Quantum chaos; semiclassical methods

PACS 42.50.Wk - Mechanical effects of light on material media, microstructures and particles

\begin{abstract}
It is shown that dynamical localization (quantum suppression of classical diffusion) in the context of ultracold atoms in periodically shaken optical lattices subjected to time-periodic modulations having equidistant zeros depends on the impulse transmitted by the external modulation over half-period rather than on the modulation amplitude. This result provides a useful principle for optimally controlling dynamical localization in general periodic systems, which is capable of experimental realization.
\end{abstract}

Copyright (C) EPLA, 2015

Introduction. - Quantum effects in transport phenomena in classical systems represent an interesting fundamental issue in quantum theory that started from a remark of Einstein in his celebrated paper on torus quantization [1]. One of such effects, widely studied in the context of time-periodic systems, is the quantum suppression of classical chaotic diffusion [2-7], or dynamical localization (DL) for short. Remarkably, this effect is a quantum manifestation of the fact that a time-periodic modulation can stabilize a system, and it is thus expected to play a key role in our understanding of the problem of quantum-classical correspondence in classically chaotic systems $[8,9]$. While it is natural to think that, with the period fixed, this effect must depend on the temporal rate at which energy is transferred from the driving mechanism to the system, i.e., on the modulation waveform, the main target of study up to now has only been their dependence on the modulation amplitude because of the traditional use of sinusoidal modulation. Recent work has provided strong evidence for a different dependence of DL on sinusoidal and square-wave modulations [10]. Since there are infinitely many different waveforms, a natural question arises: How can the influence of the shape of a periodic modulation on DL be quantitatively characterized. In this work, we demonstrate that for space-periodic systems subjected to a generic AC time-periodic modulation with equidistant zeros such characterization is well provided by a single quantity: the impulse transmitted by the modulation over half-period - hereafter called modulation impulse. This impulse is a quantity that accounts simultaneously for the modulation's amplitude, period and waveform.

Theoretical approach. - The dynamics of our model system of ultracold atoms interacting with a phasemodulated light field produced using an oscillating mirror is well described by the periodic Hamiltonian

$$
\widetilde{H}=\widetilde{p}^{2} /(2 M)-V_{0} \cos [2 k \widetilde{x}-\lambda F(t)],
$$

where $M$ is the atomic mass, $\widetilde{x}$ the position, $\widetilde{p}$ the momentum, $V_{0}$ the potential height, $k$ the wave number, $\lambda$ the dimensionless modulation depth, and $F(t)$ the AC modulation given by

$$
F(t)=F(t ; m, T) \equiv N(m) \operatorname{sn}[4 K t / T] \operatorname{dn}[4 K t / T],
$$

where $\operatorname{sn}(\cdot) \equiv \operatorname{sn}(\cdot ; m)$ and $\operatorname{dn}(\cdot) \equiv \operatorname{dn}(\cdot ; m)$ are Jacobian elliptic functions of parameter $m$, and $K \equiv K(m)$ is the complete elliptic integral of the first kind. $N(m)$ is the 

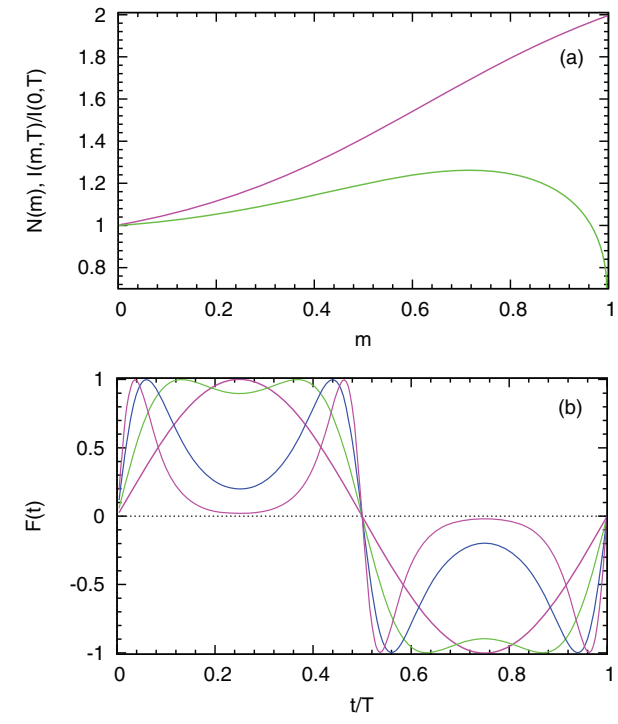

Fig. 1: (Colour on-line) (a) Normalization function $N(m)$, given by eq. (3), vs. $m$ (top pink line), and normalized modulation impulse, $I(m, T) / I(0, T) \equiv N(m) K(0) /[N(0) K(m)]$, given by eq. (4), vs. $m$ (bottom green line). (b) Modulation $F(t)$, given by eq. (2), vs. $t / T$, being $T$ the period, for four values of the shape parameter: $m=0$ (red sinusoidal pulse), $m=0.72$ (green nearly square-wave pulse), $m=0.99$ (blue double-humped pulse), and $m=0.999999$ (pink sharp doublehumped pulse).

normalization factor shown in fig. 1(a), and given by

$$
N(m) \equiv\left\{a+\frac{b}{1+\exp [(m-c) / d]}\right\}^{-1},
$$

where the values of the parameters are set equal to $a \equiv 0.43932, b \equiv 0.69796, c \equiv 0.3727$, and $d \equiv 0.26883$, in order to have the same modulation amplitude (equal to unity) and period, $T$, independently of the waveform, i.e. $\forall m \in[0,1]$, as shown in fig. 1(b). Notice that $F$ in (1) introduces a convenient (since allowing obtaining many analytical results, see eq. (13) below) flexible periodic pulse which varies its form depending on the value of $m$. For $m=0$, one recovers the well-known harmonic excitation case previously considered for example in refs. [5-7], since then $F(t ; m=0, T)=\sin (2 \pi t / T)$. On the other hand, for $m \neq 0$ the waveform has different shapes. For example, for $m=0.72$ a nearly square-wave pulse is obtained, whereas for the limiting value $m=1$ the modulation vanishes.

As will be shown below, the modulation impulse associated with $F(t)$, defined as

$$
I \equiv I(m, T)=\int_{0}^{T / 2} F(t ; m, T) \mathrm{d} t=\frac{T N(m)}{2 K(m)},
$$

is a relevant quantity to characterize the effect of the modulation's waveform. As expected, it is a function of $m$, which has a single maximum at $m=m_{\max }^{I} \simeq 0.717$, as shown in fig. 1(a). Also, it tends to zero very quickly as $m \rightarrow 1$.

Next, a few comments regarding the model represented by eq. (1) are in order. First, it is important to note that we use a single-particle Hamiltonian due to the fact that ultracold atoms in a periodically shaken optical lattice are essentially noninteracting if the ensemble is sufficiently dilute. Second, for large enough detunings, the atomic hyperfine structure does not need to be taken into account since the probability of spontaneous emission of a photon is negligible. Third, since Hamiltonian (1) is onedimensional, only optical lattices where the confining in two of the spatial directions is much narrower than in the remaining one can be described [11]. Fourth, our model does not take into account the ratchet effect, i.e. the directed transport due to symmetry breaking of zero-mean forces [12]. Nevertheless, it has been recently shown that optimum enhancement of ratchet transport is achieved when maximal effective (critical) symmetry breaking occurs, which is in turn a consequence of two reshapinginduced competing effects: i) the increase in the degree of symmetry breaking, and ii) the decrease in the (normalized) transmitted modulation impulse $[13,14]$, thus confirming the general relevance of this quantity.

Switching now to scaled dimensionless variables

$$
\tau \equiv \omega t, \quad x \equiv 2 k \widetilde{x}, \quad p \equiv \frac{2 k}{M \omega} \widetilde{p}
$$

the Hamiltonian in eq. (1) is transformed into the following dimensionless one:

$$
H \equiv \frac{4 k^{2}}{M \omega^{2}} \widetilde{H}=\frac{p^{2}}{2}-\kappa \cos [x-\lambda F(\tau ; m)],
$$

where $\kappa \equiv V_{0} k^{2} T^{2} /\left(\pi^{2} M\right), F(\tau ; m) \equiv N(m) \operatorname{sn}(\Omega \tau)$ $\operatorname{dn}(\Omega \tau)$, and $\Omega \equiv 2 K(m) / \pi$. By rewriting eq. (6) in the form $H=H_{0}+H_{1}$, with

$$
\begin{aligned}
& H_{0} \equiv \frac{p^{2}}{2}-\kappa \cos x \\
& H_{1} \equiv \kappa\{\cos x-\cos [x-\lambda N(m) \operatorname{sn}(\Omega \tau) \operatorname{dn}(\Omega \tau)]\}
\end{aligned}
$$

$H$ can be regarded now as the Hamiltonian of an effective perturbed pendulum, being $H_{1}$ the perturbation for $\lambda>0$, such that expression

$$
\frac{\mathrm{d} H_{0}}{\mathrm{~d} \tau}=\kappa p\{\sin x-\sin [x-\lambda N(m) \operatorname{sn}(\Omega \tau) \operatorname{dn}(\Omega \tau)]\}
$$

accounts for the effect of the perturbation on $H_{0}$. It has been shown for the cases of sinusoidal $[5,6]$ and squarewave [10] modulations that the strength of the DL of system (1) is correlated with the chaotic layer width of the aforementioned perturbed pendulum. For the present case, one can obtain an analytical estimate of the chaotic layer width for the case of small modulation amplitude such that

$$
\sin [x-\lambda F(\tau ; m)] \approx \sin x-\lambda F(\tau ; m) \cos x
$$


by calculating the change of the averaged energy along the separatrix

$$
\begin{aligned}
& x_{0, \pm}(\tau)= \pm 2 \arctan \left\{\sinh \left[\sqrt{\kappa}\left(\tau-\tau_{0}\right)\right]\right\}, \\
& p_{0, \pm}(\tau)= \pm 2 \sqrt{\kappa} \operatorname{sech}\left[\sqrt{\kappa}\left(\tau-\tau_{0}\right)\right]
\end{aligned}
$$

of the unperturbed pendulum $H_{0}$

$$
\left\langle\frac{\mathrm{d} H_{0}}{\mathrm{~d} \tau}\right\rangle \equiv \int_{-\infty}^{\infty} \frac{\mathrm{d} H_{0}}{\mathrm{~d} \tau} \mathrm{d} \tau
$$

where $\tau=\tau_{0}$ is an arbitrary initial time [15]. After some simple algebraic manipulation, one straightforwardly obtains

$$
\begin{aligned}
& \max _{\tau_{0}}\left\langle\mathrm{~d} H_{0} / \mathrm{d} \tau\right\rangle \approx d(\lambda, \kappa, m)+\mathcal{O}\left(\lambda^{2}\right) \\
& d \equiv d(\lambda, \kappa, m)=\frac{4 \pi^{3} \lambda N(m)}{\kappa \sqrt{m} K^{2}(m)} \sum_{n=0}^{\infty} a_{n}(\kappa) b_{n}(m)
\end{aligned}
$$

where

$$
\begin{aligned}
& a_{n}(\kappa) \equiv(n+1 / 2)^{3} \operatorname{sech} \frac{(n+1 / 2) \pi}{\sqrt{\kappa}}, \\
& b_{n}(m) \equiv \operatorname{sech} \frac{(n+1 / 2) \pi K(1-m)}{K(m)} .
\end{aligned}
$$

The width function $d(\lambda, \kappa, m)$ gives a first-order approximation in $\lambda$ to the width in energy of the chaotic separatrix layer. As a function of $m$, it presents a single maximum at $m=m_{\max }^{d} \simeq 0.651$, which is significantly near $m_{\max }^{I} \simeq 0.717$, in the sense that the respective waveforms are almost coincident (see discussion below). Also, $\lim _{m \rightarrow 1} d(\lambda, \kappa, m)=0$, as the modulation vanishes in that limit irrespectively of the (finite) value of $\lambda$. Thus, one would expect the dependence of the width function on the shape parameter to remain approximately (qualitatively) valid for values of the dimensionless modulation depth which are beyond the first-order perturbative regime.

Numerical results. - In this section we compare the classical and quantum dynamics of our system using different tools. First, by calculating the momentum distributions, we identify the classical footprint of DL in the quantum dynamics. Second, to further demonstrate the influence of the classical phase space structures in the quantum behavior of the system, we compare the (classical) Poincaré surfaces of section (PSOS) and the corresponding Husimi-based quantum surfaces of section (QSOS) [16], obtaining an excellent concordance between the results yielded by both tools.

Momentum distributions. The classical momentum distribution for our system is numerically calculated as

$$
P_{C}(p, \tau)=\int P(x, p ; \tau) \mathrm{d} x,
$$

where $P(x, p ; \tau)$ is the evolution of a uniform distribution over one wavelength with a Gaussian momentum distribution characterized by a width of $\Delta p_{0}=0.386$ [4-6] given by the corresponding Liouville equation, defined as

$$
\left(\partial_{\tau}+p \partial_{x}-\kappa \sin [x-\lambda F(\tau ; m)]\right) P(x, p ; \tau)=0,
$$

subjected to periodic boundary conditions $P(x+L, p ; \tau)=P(x, p ; \tau), L=2 n \pi$, with $L$ being the size of the quantization box and $n \in \mathbf{Z}^{+}$. Equation (16) is integrated using a standard fourth-order Runge-Kutta method [17].

On the other hand, quantum-mechanical momentum distributions, $P_{Q}(p, \tau)$, were computed by averaging time propagations with the time-dependent Schrödinger equation

$$
i \hbar_{\mathrm{eff}} \partial_{\tau} \psi=-\left\{\frac{\hbar_{\mathrm{eff}}^{2}}{2} \partial_{x x}^{2}+\kappa \cos [x-\lambda F(\tau ; m)]\right\} \psi
$$

solved using a fast Fourier transform method [17] for the localized wave packets

$$
\psi(x, t)=\left(\pi \Delta x_{0}\right)^{-1 / 4} \exp \left[-\frac{\left(x-x_{0}\right)^{2}}{2 \Delta x_{0}}+\frac{i x p_{0}}{\hbar_{\mathrm{eff}}}\right],
$$

centered at $x_{0} \in[0, L]$ with $p_{0}=0$ and $\Delta x_{0}=\hbar_{\mathrm{eff}} / \Delta p_{0}$, where $\hbar_{\mathrm{eff}} \equiv 2 \hbar k^{2} T /(\pi M)$ is the effective Planck constant characterizing the "quanticity" degree of the system.

The strength of DL is quantified by the difference between the classical and quantum-mechanical momentum distributions $\Delta p_{C-Q} \equiv \Delta p_{C}-\Delta p_{Q}$, being

$$
\Delta p_{i} \equiv \sqrt{\left\langle p_{i}^{2}\right\rangle-\left\langle p_{i}\right\rangle^{2}}, \quad i=\{C, Q\},
$$

the normalized root-mean-square momentum widths. Recall that the larger $\Delta p_{C-Q}$, the stronger the DL.

In order to demonstrate that the modulation impulse is a relevant quantity properly controlling DL, we show in fig. 2 the classical, $\Delta p_{C}$, and quantum, $\Delta p_{Q}$, momentum distributions as a function of $\lambda$, as well as the difference, $\Delta p_{C-Q}$, for four different values of $m$, i.e. four different modulation waveforms. As it can be seen, the numerical results confirm that one needs larger values of the modulation amplitude $\lambda$ to obtain a noticeable DL as the modulation impulse (as a function of $m$ ) decreases from its maximum value at $m=m_{\max }^{I} \simeq 0.717$.

In fig. 3(a), we show the difference between the classical and the quantum momentum distributions, $\Delta p_{C-Q}$, as a function of the shape parameter, $m$, for $\hbar_{\mathrm{eff}}=0.16$ and 0.30 , keeping the rest of the parameters $(\kappa, \lambda)$ fixed. In this figure, we have superimposed the chaotic layer width in energy (4) and the modulation impulse. As it is shown, $\Delta p_{C-Q}$ presents its maximum values over a range $0.65 \leq m \leq 0.7$, which is close to the impulse maximum $m_{\max }^{I}$ (cf. fig. 1(a)). Although not shown in fig. 3 , the four plotted quantities vanish in the limit $m \rightarrow 1$. Moreover, it is also important to remark the excellent agreement that exists between the chaotic layer width and the modulation impulse, that nearly coincide when adequately 

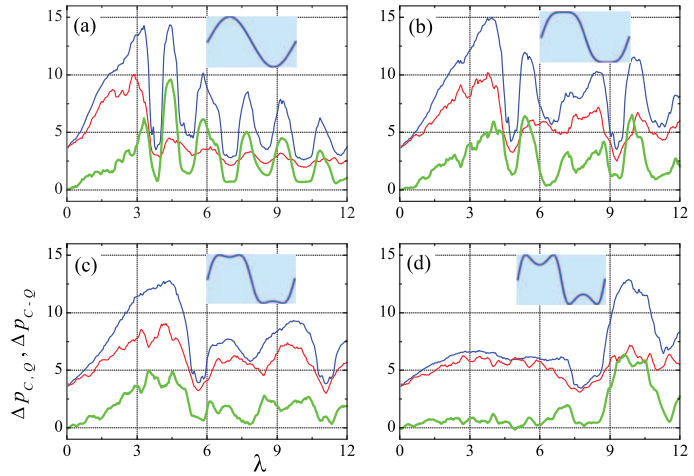

Fig. 2: (Colour on-line) Comparison of the classical- (thin blue lines), $\Delta p_{C}$, and quantum-mechanical (medium red lines), $\Delta p_{Q}$, momentum distributions of atoms, and their differences (thick green lines), $\Delta p_{C-Q}$, as functions of the modulation amplitude, for $\kappa=0.36, \hbar_{\mathrm{eff}}=0.16$ and four modulation waveforms: (a) $m=0$, (b) $m=0.5$, (c) $m=0.7$, and (d) $m=0.9$. Insets: wave forms over a modulation period.

scaled. Thus, we can unambiguously conclude the correlation existing between these two quantities characterizing, respectively, the degree of chaoticity of the system and the AC modulation.

In fig. 3(b), we show the difference between the classical and the quantum momentum distributions, $\Delta p_{C-Q}$, as a function of $\hbar_{\mathrm{eff}}$ for different values of $m$. As can be seen, the function $\Delta p_{C-Q}$ increases monotonically for small values of $\hbar_{\mathrm{eff}}$, up to $\hbar_{\mathrm{eff}} \approx 0.6$, where it saturates remaining practically constant. Consequently, $\Delta p_{C-Q}$ is well fitted using an exponential function, as shown in the figure for $m=0.717$. Notice also that the top curve of $\Delta p_{C-Q}$ is associated with $m_{\max }^{I}$, which agrees remarkably well with the results shown in fig. 3(a).

To further demonstrate the influence of the modulation waveform on DL, we show PSOSs for three different representative values of $m$ in fig. 4 (top panel). These PSOSs have been stroboscopically computed at times multiples of the modulation period $T=2 \pi$. One sees that the modulation waveform, and hence the modulation impulse, does not only have a great influence on the DL strength, but also on the phase space structures of the system. Thus, for $m=0$ - a value of $m$ for which DL clearly occurs - there are only very tiny islands of regularity (leftmost panel), which are drastically reduced over the range of maximum strength of $\mathrm{DL}, m_{\max }^{I}=0.717$ (central panel). Finally, for values of $m$ which are sufficiently close to 1 , as for $m=0.9999$ (rightmost panel), DL is very weak, while the area occupied by regularity islands becomes even larger as $m \rightarrow 1$. Clearly, this confirms the predicted DL scenario.

Influence of the modulation waveform on the quantum dynamics. In this section we show how the quantum dynamics of our system, as it happens in general, is dramatically influenced by the underlying classical structures and, as a consequence, by the modulation waveform. To demonstrate this effect, we calculate QSOSs for some representative values of the shape parameter $m$. Similarly to
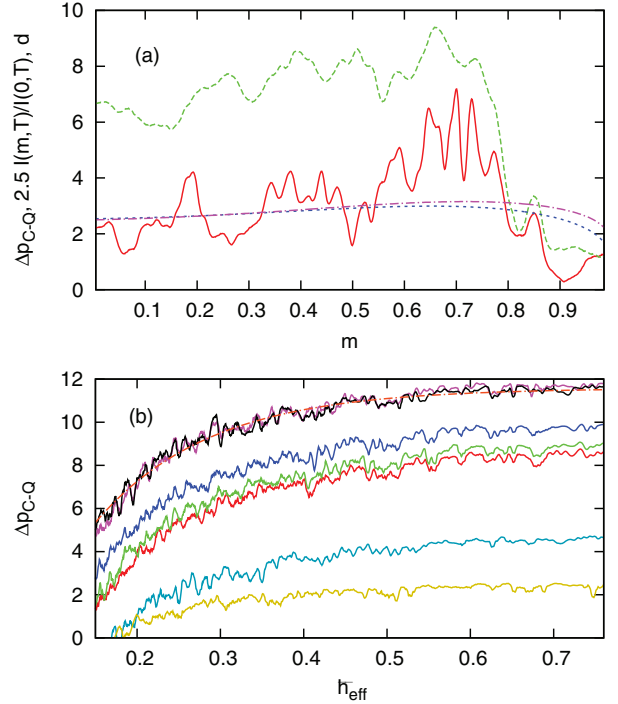

Fig. 3: (Colour on-line) Difference between the classicaland quantum-mechanical momentum distributions, $\Delta p_{C-Q}$, for $\kappa=0.36$ and $\lambda=2$. (a) $\Delta p_{C-Q}$ for $\hbar_{\mathrm{eff}}=0.16$ (continuous red line) and $\hbar_{\text {eff }}=0.30$ (long-dashed green line), width of the chaotic layer, $d$, given by eq. (13) (short-dashed blue line), and normalized modulation impulse, $2.5 I(m, T) / I(0, T)$, given by eq. (4) (dashed-dotted pink line) vs. the shape parameter $m$. (b) $\Delta p_{C-Q}$ vs. $\hbar_{\mathrm{eff}}$ for $m=0$ (red line), 0.2 (green line), 0.4 (dark blue line), 0.6 (pink line), 0.717 (black line), 0.8 (light blue line), 0.99999 (bottom yellow line), along with the fitting function $\Delta p_{C-Q}\left(\hbar_{\mathrm{eff}}\right)=11.6-18.6 \exp \left(-7.3 \hbar_{\mathrm{eff}}\right)$ for $m_{\max }^{I}=0.717$ (dashed-dotted orange line).

the classical PSOSs, we stroboscopically computed these QSOSs at multiples of the modulation period, $T=2 \pi$, using the Husimi quasi-probability distribution [16]

$$
\mathcal{H}(x, p, t)=\left|\int_{-\infty}^{\infty} \exp \left[-\frac{(x-z)^{2}}{2 \alpha}-i \frac{z p}{\hbar_{\mathrm{eff}}}\right] \psi(z, t) \mathrm{d} z\right|^{2},
$$

with $\alpha=3$. This expression can be rewritten as a sum of infinite terms, due to the existing periodic boundary conditions, as

$$
\begin{aligned}
\mathcal{H}(x, p, t)= & \mid \sum_{n=-\infty}^{\infty} \int_{0}^{2 \pi} \exp \left\{-\frac{[x-(z+2 \pi n)]^{2}}{2 \alpha}\right. \\
& \left.-i \frac{(z+2 \pi n) p}{\hbar_{\mathrm{eff}}}\right\}\left.\psi(z+2 \pi n, t) \mathrm{d} z\right|^{2},
\end{aligned}
$$

although the calculation is effectively limited to the first three or four terms due to the Gaussian decay. By averaging the QSOS over the times used in the calculation of the corresponding classical PSOS, we can quantummechanically unfold the phase space structure of the system, which consists of a mix of islands of regularity and regions of stochasticity. Moreover, this averaged QSOS can be directly compared with the corresponding classical PSOS. Some results for different representative values of the parameter $m$ are shown in fig. 4 (bottom panels), where the QSOSs after averaging over 50 

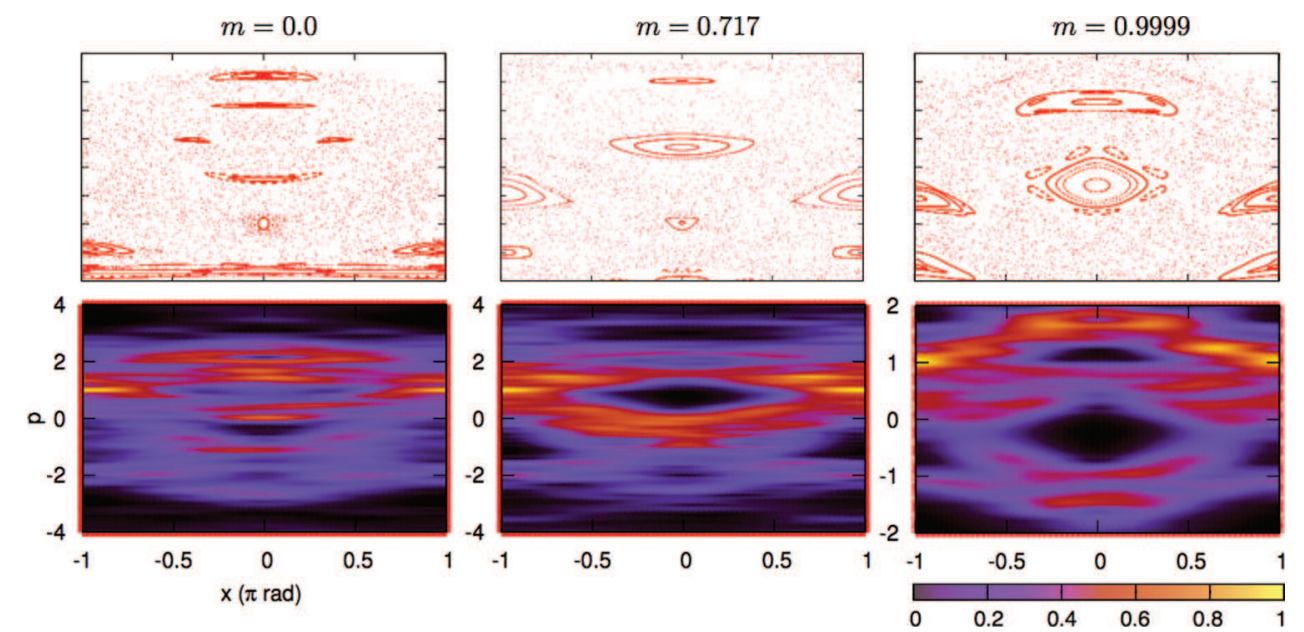

Fig. 4: (Colour on-line) Poincaré surfaces of section (top) and Husimi-based quantum surfaces of section (bottom) obtained from the wave function given by eq. (18) with $x_{0}=\pi$ and $p_{0}=1$ averaged stroboscopically over 50 time periods for $m=0$ (left), $m=m_{\max }^{I}=0.717$ (center), and $m=0.9999$ (right).

modulation periods for a coherent state (18) centered at $\left(x_{0}, p_{0}\right)=(\pi, 1)$ are depicted. This initial condition gives rise to a classically chaotic trajectory. One sees that the three QSOSs appear all spread out over the corresponding chaotic regions of phase space. We also notice how the probability density does not enter into the regions where the motion is regular, since the corresponding tori and resonant islands act as total barriers both for the classical motion and the flow of quantum probability across. This effect is clearly more noticeable as $m$ is increased, i.e. when larger portions of phase space become regular. As a consequence, the QSOSs mimic to a great extent the corresponding classical PSOSs. One point is worth mentioning here. If we had propagated the initial wave functions for a much longer time, the corresponding QSOSs would have entered to some extent inside these regions of regularity due to the usual quantum tunneling. For a more detailed description of the Husimi time evolution corresponding to the case $m=0.9999$ of fig. 4 see the supplementary video in ref. [18].

Let us consider now the quantum dynamics associated with other types of classical motion. When the wave function is initially localized at a point associated with a regular classical trajectory, whose dynamics is then confined to the corresponding invariant tori or chain of islands, the quantum quasi-probability density behaves accordingly, thus remaining well localized in the neighborhood of the tori (except, again, for very long times when quantum tunneling becomes noticeable). An example is depicted in fig. 5, where we show the QSOS corresponding to a wave function for $m=0.9999$ and $\left(x_{0}, p_{0}\right)=(0,-0.805)$. This initial condition generates a classical trajectory in the chain of islands associated with a 7:3 resonance (see the corresponding PSOS in fig. 5). One sees that the localized wave function jumps over three of the islands at the instants $t=T, 2 T, \ldots, 6 T$ and returns close (although with some accumulated delay)
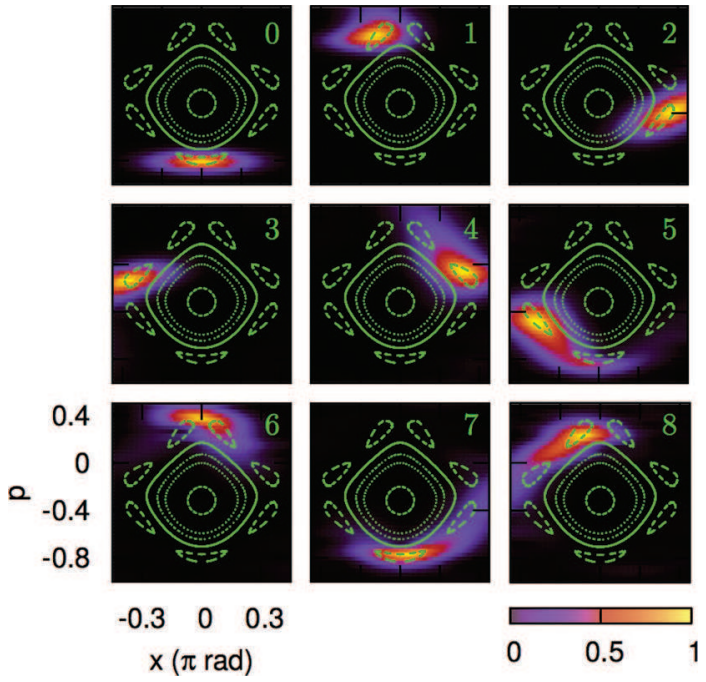

Fig. 5: (Colour on-line) Husimi-based quantum surface of section (21) for the wave function (18) launched at $\left(x_{0}, p_{0}\right)=$ $(0,-0.805)$ for $m=0.9999$. The corresponding classical Poincaré surface of section has been represented as superimposed green dots. The number at the top right corner of each panel indicates the elapsed time in units of the modulation period $T=2 \pi$.

to the initial position at $t=7 T$ (see the supplementary video in ref. [18] for the evolution at intermediate instants). Note that this is the behavior expected from the dynamics associated with the $7: 3$ classical resonance.

It is worth noting that if we add all the QSOSs distributions shown in fig. 5 and renormalize the total, we get a new distribution highly localized on the corresponding classical PSOS. This total distribution is depicted in fig. 6(a), where the described effect is clearly visible. Figures 6(b)-(f) show similar results for QSOS localized on other relevant regular structures existing in the classical 


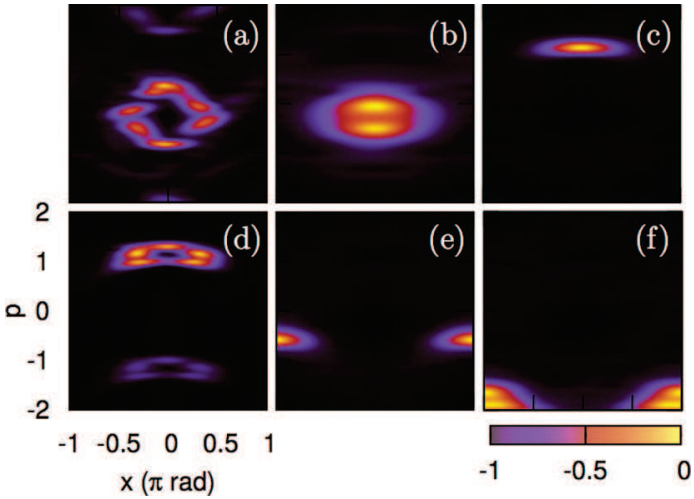

Fig. 6: (Colour on-line) Long-time averaged Husimi-based quantum surfaces of section corresponding to wave function (18) initially launched at different points in phase space for $m=0.9999$. All the panels have been (stroboscopically) averaged over 50T, except panels (a) and (d), which have been averaged over $7 T$ and $5 T$, respectively.

PSOS of fig. 4 for $m=0.9999$. For this purpose, we have used wave functions launched at suitable initial conditions in phase space, and we have (stroboscopically) averaged the obtained QSOS over 50T. One sees that these averaged QSOS appear respectively localized on a torus around the central fixed point $(x, p)=(0,0)$ (fig. 6(b)), on a torus around the phase space point $(0,1)$ (fig. 6(c)), on the 5:1 chain of islands around the same point (fig. 6(d)), on a torus around the border fixed point at $( \pm \pi,-0.5)$ (fig. $6(\mathrm{e}))$, and on a torus around the other border fixed point at $( \pm \pi,-2)$ (fig. $6(f))$. In all cases, the QSOSs completely mimic the structure of the classical phase space. This clearly demonstrates the importance of this classical structures for the quantum motion. It is worth mentioning that similar results to those presented here have been obtained for other values of the shape parameter $m$.

Conclusions and final remarks. - Summarizing, in this paper it has been shown for AC-driven space-periodic Hamiltonians that the impulse transmitted by the timeperiodic modulation is an essential quantity to understand the phenomenon of DL, which can be optimally controlled by changing the form of the $\mathrm{AC}$, i.e. the parameter $m$ in eq. (2). Remarkably, our results show that the impulse principle reliably control DL irrespectively of the "quanticity" degree of the system. While this result holds for the wide class of AC modulations having equidistant consecutive zeros, one expects it to be also valid for an even more general class of time-periodic modulations, at least in the adiabatic regime [19]. This principle, which can be straightforwardly applied to other phenomena, such as field-induced barrier transparency [20] or quasi-energy band collapse [21], paves the way for optimum coherent control of diverse quantum systems. Additionally, it has been shown that the classical invariant structures of phase space have a deep impact on the quantum-dynamical behavior of the system, as the modulation impulse is varied.

$$
* * *
$$

This research has been supported by the Ministry of Economy and Competitiveness (MECC, Spain) under Contract Nos. MTM2012-39101, FIS2012-34902, and ICMAT Severo Ochoa SEV-2011-0087, and Junta de Extremadura (JEx, Spain) though project No. GR10045.

\section{REFERENCES}

[1] Einstein A., Dtsch. Phys. Ges. Verh., 19 (1917) 82.

[2] Casati G., Chirikov B. V., Izrailev F. M. and Ford J., Lect. Notes Phys., 93 (1979) 334.

[3] Fishman S., Grempel D. R. and Prange R. E., Phys. Rev. Lett., 49 (1982) 509.

[4] Moore F. L., Robinson J. C., Bharucha C., Williams P. E. and Raizen M. G., Phys. Rev. Lett., 73 (1994) 2974.

[5] Graham R., Schlautmann M. and Zoller P., Phys. Rev. A, 45 (1992) R19.

[6] Chacón R., EPL, 77 (2007) 30001.

[7] Abal G., Donangelo R., Romanelli A., SicardiSchifino A. C. and Siri R., Phys. Rev. E, 65 (2002) 046236.

[8] Reichl L. E., The Transition to Chaos in Conservative Classical Systems: Quantum Manifestations (SpringerVerlag, New York) 1992.

[9] Casati G. and Chirikov B. V. (Editors), Quantum Chaos between Order and Disorder (Cambridge University Press, Cambridge) 1995.

[10] Chacón R., Borondo F. and Farrelly D., EPL, 86 (2009) 30004.

[11] Bharucha C. F., Robinson J. C., Moore F. L., Sundaram B., Niu Q. and Raizen M. G., Phys. Rev. E, 60 (1999) 3881.

[12] Hänggi P. and Marchesoni F., Rev. Mod. Phys., 81 (2009) 387.

[13] Chacón R., J. Phys. A, 40 (2007) F413.

[14] Chacón R., J. Phys. A, 43 (2010) 322001.

[15] Lichtenberg A. J. and Lieberman M. A., Regular and Chaotic Dynamics (Springer-Verlag, Berlin) 1991.

[16] Husimi K., Proc. Phys. Math. Soc. Jpn., 22 (1940) 264.

[17] Press W. H., Teukolsky S. A., Vetterling W. T. and Flannery B. P., Numerical Recipes: The Art of Scientific Computing, third edition (Cambridge University Press, Cambridge) 2007.

[18] See the supplementary video at http://youtu.be/ E03vnhcjMKQ for the time evolution of the Husimi distribution of a Gaussian wave packet initially centered at a point associated to a chaotic and a regular trajectory.

[19] Chacón R., Uleysky M. Yu. and Makarov D. V., EPL, 90 (2010) 40003.

[20] Vorobeichik I., Lefebvre R. and Moiseyev N., Europhys. Lett., 41 (1998) 111.

[21] Dunlap D. H. and Kenkre V. M., Phys. Rev. B, 34 (1986) 3625. 